Editorial

Wien klin Mag 2017 · 20:195

https://doi.org/10.1007/s00740-017-0204-z

(c) Springer-Verlag GmbH Austria, ein Teil von Springer Nature 2017

CrossMark

Verena Kienast

SpringerMedizin, Wien, Österreich

\title{
Der schwierige Umgang mit Fehlern
}

\section{Plädoyer für kleine Schritte zu einer offenen Fehlerkultur}

Fehler, Fehlentwicklungen und unangenehme Erlebnisse zu verschweigen, kann gefährlich werden. Zum einen lässt sich meist die Folgewirkung dadurch nicht verhindern, zum anderen wird die Ursache des Fehlers nicht beseitigt und es werden möglicherweise weitere Fehler passieren. Dieser Überlegung folgend, haben sich vor allem in der Berufswelt Systeme etabliert, um die Meldung von Fehlern bzw. unerwünschten Entwicklungen an verantwortliche Stellen $\mathrm{zu}$ melden. Denn nicht immer ist das Umfeld erfreut, wenn darüber gesprochen wird, wenn Gruppeninteressen torpediert werden. Der „Whistle blower", also der „Aufdecker“ aus den eigenen Reihen, übernimmt eine zwiespältige Rolle: Er meldet, was ihm falsch erscheint - und stellt damit die Beteiligten an den Pranger. In der Gruppe wird er sich damit kaum beliebt machen und seine zukünftige Position wird sich wahrscheinlich verändern.

\section{Zwischen Aufdeckung und Denunziation}

Gleichzeitig erhöht sich mit der Förderung von „Fehlermeldungen“ durch Organisationsleitungen das Risiko, dass es nicht mehr so sehr um den Fehler/die Fehlentwicklung/das Ereignis geht, sondern eigennützige Interessen solche Meldungen steuern. Man könnte unliebsame Kollegen in ein schlechtes Licht stellen und damit selbst besser dastehen oder eine begehrte Stelle selbst bekommen. Ein gewisses Maß an Eitelkeit lässt sich bei manchem öffentlichkeitswirksamen „Whistle Blower“ wohl nicht bestreiten, gleichzeitig ist die Essenz der Aufdeckung in den meisten Fällen durchaus würdig, beanstandet $\mathrm{zu}$ werden und bedarf der Änderung/Verbesserung. Und der Mut eines Einzelnen, das auszusprechen, worüber viele andere lieber schweigen oder bisher nicht den Mut hatten zu sprechen, kann in der Folge auch die weniger Mutigen dazu veranlassen, ihre Sicht der Dinge offen zu legen. Wie Organisationen mit diesen Informationen und mit den Überbringern dieser Informationen umgehen, ist eine Frage der Verantwortung und des Fingerspitzengefühls. Denn die Grenze zur Denunziation kann unscharf sein.

\section{Nicht immer ist es eine böse Absicht}

Fehlermeldesysteme wie das CIRS - Critical Incident Report System, das sowohl in der Luftfahrt als auch im Gesundheitswesen zum Einsatz kommt, sind anonymisiert und Meldungen werden auf ihre Glaubwürdigkeit und Relevanz geprüft. So können Verbesserungen ohne persönliche Schuldzuweisungen in die Wege geleitet und umgesetzt werden. Und im Idealfall die dauerhafte Umsetzung begleitend evaluiert. Nicht jeder Fehler schleicht sich schließlich mit böser Absicht ein, aber gewohnte Abläufe haben ein gewisses Beharrungsvermögen. Die heuer von der Plattform Patientensicherheit in den Mittelpunkt der Aktivitäten gestellte Initiative „Speak up“ soll auf allen Seiten das Bewusstsein dafür stärken, Dinge auszusprechen, die nicht richtig erscheinen. Gleichzeitig müssen die Adres- saten im Gesundheitssystem bereit sein, diese Beobachtungen und Kritik ernst zu nehmen. Das ist nicht immer einfach. Vor allem dann nicht, wenn man derartige Mitteilungen persönlich nimmt bzw. wenn diese auf einer persönlich verletzenden Ebene abgegeben werden.

Auch der Umgang mit Fehlern kann gelernt werden. Sachlichkeit und Respekt bewahren vor einem übermäßig emotionalen Umgang und die Schuldfrage sollte endlich der Frage nach zielführenden Lösungen weichen. Kleine Schritte zu einer offenen Fehlerkultur sind jedenfalls besser als der verbissene Anspruch darauf, Recht zu haben. Um das geht es nämlich gar nicht

meint Ihre

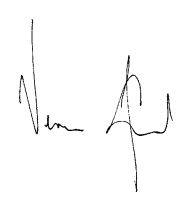

\section{Korrespondenzadresse}

\section{Kienast}

SpringerMedizin Wien, Österreich

Verena.kienast@springer.at 